K.A. van der Hucht, G. Koenigsberger \& P.R.J. Eenens, eds.

\title{
Discovery of $50000 \mathrm{~K}$ shocked gas in NGC 6888
}

\author{
Reginald J. Dufour and Brent Buckalew \\ Rice University, Houston, TX 77005, USA
}

\begin{abstract}
We report the results of a re-analysis of KPNO-IRS spectroscopy of 30 locations in the prototypical WR shell nebula NGC 6888 (WN6 central star). The spectra covered a $3500-7200 \AA$ wavelength range with $R \simeq 360$ effective spectral resolution. Electron temperatures for a majority of the locations were derived from the [N II] $\lambda \lambda(6548+6583) / 5755$ and the [O III] $\lambda \lambda(4959+5007) / 4363$ emission line ratios. $T_{\mathrm{e}}([\mathrm{N} \mathrm{II}]) \simeq 8000 \pm 2400 \mathrm{~K}$ for eleven locations in the north and west parts of the shell and $T_{\mathrm{e}}([\mathrm{N} \mathrm{II}]) \simeq 13000 \pm 4000 \mathrm{~K}$ for five locations in the southwest part. By contrast, the [O III] temperatures show great variation with location, ranging from $12000 \mathrm{~K}$ to $55000 \mathrm{~K}$, with the highest value being that for an [O III] filament located to the north and outside of the ensemble of knots defining the northern extent of the mass loaded ejecta from the star.

Using a combination of spectral 'arithmetic', shock- and photoionization models, we demonstrate that NGC 6888 is permeated by a strong hot windshocked gaseous medium (which extends outside the mass-loaded ejected material in the north and west) that contaminates the spectrum of any given location and must be removed to determine realistic $T_{\mathrm{e}}, N_{e}$, and abundances for the ejected material in the observed knots. For some knots, the effects are such that the abundances obtained via naive empirical methods were found to be several times too small (particularly for $\mathrm{O} / \mathrm{H}$ ) than in reality. We present a few illustrative examples and discuss the implications the results have on abundances previously derived for NGC 6888, but more importantly, what these results imply about the origin of temperature fluctuations in other types of nebulae which may be influenced by stellar winds (i.e., planetary nebulae and $\mathrm{H}$ II regions).
\end{abstract}

\section{Introduction}

The observations consist of CCD imagery taken with the Palomar 60-inch telescope through interference filters isolating various strong emission lines and spectroscopy with the KPNO $0.9 \mathrm{~m}$ and IRS spectrograph of 30 locations selected on the basis of ionization variations evident from the imagery. Details of the observations, reductions, and initial analyses can be found in Mitra (1990).

We re-measured selected emission lines in the IRS spectra of locations in NGC 6888, which showed the auroral lines of [O III] $\lambda 4363$ and/or [N II] $\lambda 5755$, and derived new electron temperatures using the NEBULAR.STSDAS software developed by Shaw \& Dufour (1995) incorporating modern atomic data.

\section{Results and implications}

Altogether, we measured $T_{\mathrm{e}}$ from [N II] at 12 locations and $T_{\mathrm{e}}$ from [O III] at 10 locations, with 8 locations in common. In all of the common locations was $T_{\mathrm{e}}[\mathrm{N} \mathrm{II}] \leq T_{\mathrm{e}}[\mathrm{O} \mathrm{III}]$ by amounts ranging from $\sim 3000 \mathrm{~K}$ to over $\sim 20000 \mathrm{~K}$ for 

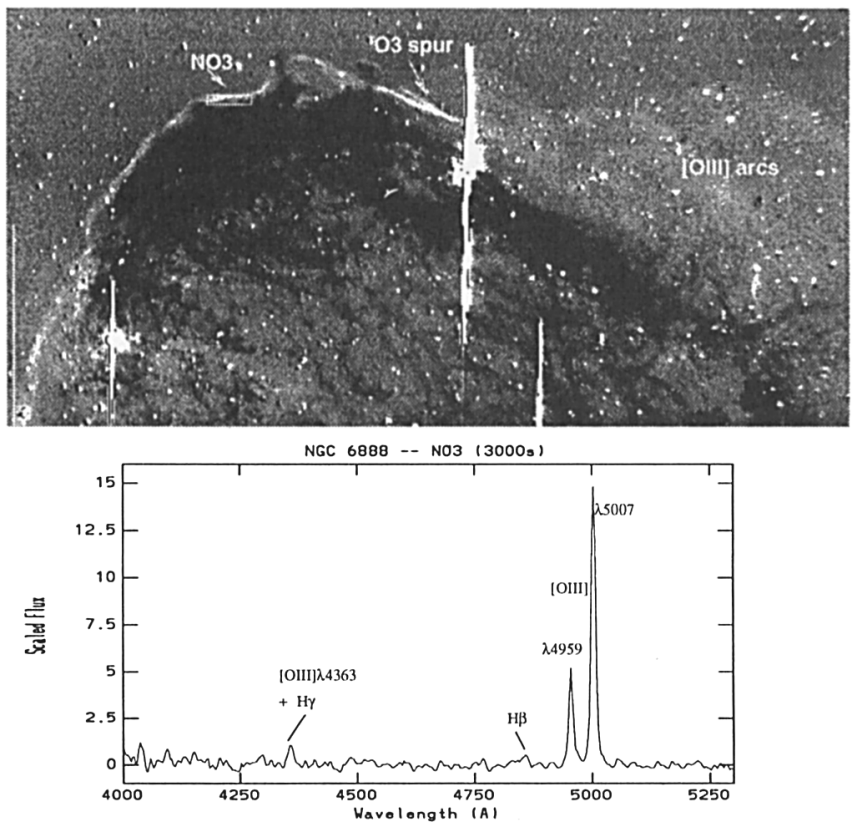

Figure 1. Top: Image of the northern part of NGC 6888 made by subtracting CCD images in [O III] (coded white) and $\mathrm{H} \alpha$ (coded black). Bottom: Part of the KPNO-IRS spectrum of the NO3 filament showing the dominance of the [O III] lines compared to hydrogen Balmer lines.

locations near the northern rim of NGC 6888 where the [O III] emission extends beyond that of $\mathrm{H} \alpha$ and [N II] (cf. Fig. 1). The highest $T_{\mathrm{e}}$ measured was $55000 \pm 20000 \mathrm{~K}$ for the NO 3 location (cf. spectrum in Fig. 1).

These results indicate that the photoionized knots in NGC 6888 are permeated by high temperature wind-driven supersonic gas which affects the spectra of the knots and filaments by varying degrees. Therefore, this hot component must be subtracted out of the spectra of the knots in order to derive accurate abundances. In addition, such a differing mix of plasmas results in large temperature fluctuations $\left(t^{2} \simeq 0.5\right.$ !) at locations along the outer rim. The implication of this phenomenon, so evident in NGC 6888, is that for planetary nebulae and $\mathrm{H}$ II regions the winds from their ionizing star(s) would produce the hot plasma responsible for significant $T_{\mathrm{e}}$ fluctuations that are observed in many.

Acknowledgments. This research was supported in part by grants NGT 2-52252 and GO-07515.02-96A to Rice University.

\section{References}

Shaw, R.A, Dufour, R.J. 1995, PASP 107, 896

Mitra, M. 1990, Ph.D. Thesis, Rice University 